\title{
Câncer gástrico e fatores de risco ambientais: As influências do regionalismo amazônico e a infecção pela Helicobacter pylori
}

\author{
Gastric cancer and environmental risk factors: The influences of amazon regionalism and \\ Helicobacter pylori infection
}

\section{Cáncer gástrico y factores de riesgo ambientales: Las influencias del regionalismo amazónico y la infección por Helicobacter pylori}

Adilson Mendes de Figueiredo Júnior ${ }^{1 *}$, Lillian Nogueira da Costa $^{1}$, João Augusto do Carmo Cardoso $^{1}$, Silvia Cristina Santos da Silva ${ }^{1}$, Elizangela Fonseca de Mendonça ${ }^{1}$, Glória Letícia Oliveira Gonçalves Lima ${ }^{1}$, Herlani Cristina Oliveira Barreto ${ }^{1}$, Suely Aleixo da Costa Sousa ${ }^{1}$, Luana Baía Menezes $^{2}$, Dionelle do Socorro Pinheiro de Oliveira ${ }^{3}$, Fabrício Tavares dos Santos ${ }^{4}$, Luísa Martins Caricio $^{4}$, Priscila Fonseca Souza ${ }^{4}$, Carla Caroline Lobo de Souza ${ }^{5}$, Mayara Melo Galvão ${ }^{5}$.

\section{RESUMO}

Objetivo: Definir os principais fatores de risco ambientais presentes nos pacientes internados em um hospital de referência em oncologia de Belém-PA, relacionando-os com peculiaridades regionais amazônicas e com a infecção pela Helicobacter pylori (H. pylori). Métodos: Trata-se de uma pesquisa com abordagem quantitativa de corte transversal, onde foram selecionados 32 pacientes em um período de 2 meses. Resultados: Observou-se como fator de risco para o adenocarcinoma gástrico idade maior que 50 anos (46,87 \% faixa etária de 51 a 60 anos e 31,37 \% faixa etária 61 a 70 anos; p - 0.0064), baixa escolaridade e renda salarial $(p-0.0016$ e $<0.0001)$, consumo de sal em alimentos processados, em especial carne seca salgada, peixe salgado e churrasco (p-0.0004, 0.0003 e 0.0064) e consumo diário de alimentos de hábitos regionais como farinha de mandioca $(p<0.0001)$ Conclusão: $A$ infecção pela $H$. pylori não foi relacionado como fator de risco importante, assim como o tabagismo e etilismo.

Palavras-chave: Fatores de risco, Neoplasias gástricas, Riscos ambientais.

\begin{abstract}
Objective: To define the main environmental risk factors present in patients hospitalized at a referral hospital in Belém-PA oncology, relating them to regional Amazonian peculiarities and to Helicobacter pylori (H. pylori) infection. Methods: This is a cross-sectional quantitative approach, where 32 patients were selected over a 2-month period. Results: A risk factor for gastric adenocarcinoma was found to be older than 50 years (46.87\% from 51 to 60 years and $31.37 \%$ from 61 to 70 years, $p=0.0064)$, low schooling and $(p-0.0016$ and $<0.0001)$, salt consumption in processed foods, especially salted dried meat, salted fish and barbecue ( $p-0.0004,0.0003$ and 0.0064$)$ and daily consumption of foods of regional habits such as cassava flour $(p<0.0001)$ Conclusion: $H$. pylori infection was not reported as an important risk factor, as was smoking and alcohol consumption.
\end{abstract}

Key words: Risk factors, Gastric neoplasms, Environmental risks.

\footnotetext{
${ }^{1}$ Escola Superior da Amazônia (ESAMAZ). Belém-Pará. *E-mail: adilsonmdfj@hotmail.com

${ }^{2}$ Faculdade Metropolitana da Amazônia (FAMAZ). Belém-Pará

${ }^{3}$ Faculdade Estácio. Castanhal-Pará

${ }^{4}$ Universidade Federal do Pará (UFPA). Belém-Pará

${ }^{5}$ Universidade do Estado do Pará (UEPA). Belém-Pará
} 


\section{RESUMEN}

Objetivo: Definir los principales factores de riesgo ambiental presentes en los pacientes internados en un hospital de referencia en oncología de Belém-PA, relacionándolos con peculiaridades regionales amazónicas y con la infección por Helicobacter pylori (H. pylori). Métodos: Se trata de una investigación con abordaje cuantitativo de corte transversal, donde fueron seleccionados 32 pacientes en un período de 2 meses.

Resultados: Se observó como factor de riesgo para el adenocarcinoma gástrico edad mayor que 50 años (46,87\% grupo de edad de 51 a 60 años y $31,37 \%$ franja de edad 61 a 70 años, $p-0.0064$ ), baja escolaridad y $(p-0.0016$ y $<0.0001)$, consumo de sal en alimentos procesados, en especial carne seca salada, pescado salado y barbacoa ( $p-0.0004,0.0003$ y 0.0064$)$ y consumo diario de alimentos de hábitos regionales como harina de mandioca Conclusión: La infección por $H$. pylori no fue relacionada como factor de riesgo importante, así como el tabaquismo y el etilismo.

Palabras clave: Factores de riesgo, Neoplasias gástricas, Riesgos ambientales.

\section{INTRODUÇÃO}

A ocorrência do câncer gástrico vem sendo associada à exposição de fatores intrínsecos decorrentes da constituição genética e a fatores extrínsecos, também denominados de fatores ambientais, como o uso de dietas com altas concentrações de cloreto de sódio, nitratos e nitritos contidos em alimentos defumados e frituras (TEXEIRA JBA, NOGUEIRA MS, 2008).

Diferenças internacionais substanciais na prevalência de doenças cancerígenas supõem que a nutrição pode ser um fator importante no desenvolvimento do câncer. Muitos especialistas acreditam que fatores nutricionais podem contribuir até $35 \%$ para o desenvolvimento de tumores malignos. Muitos pacientes perderam peso corporal substancial já no momento do diagnóstico da doença como consequência da desnutrição e desnutrição, respectivamente. Durante o curso da doença, o estado nutricional muitas vezes está se deteriorando ainda mais (IMOBERDORF R et al., 2017; MANNISTO $S$ et al., 2015; RESENDE AL et al., 2006).

Uma vez que as pessoas não consomem alimentos individuais, mas certas combinações deles, a análise dos padrões alimentares pode oferecer um aspecto adicional para avaliar as associações entre a dieta e doenças como o câncer gástrico. Também é importante examinar se as relações entre padrões alimentares e risco de câncer são consistentes entre as variadas populações. É de fundamental importância o estudo dos padrões dietéticos e risco de câncer enfatizando as variabilidades regionais (MANNISTO S et al., 2015).

A ocorrência do câncer gástrico vem sendo muito associada à exposição de fatores, principalmente, dietéticos, estes que incluem dietas com altas concentrações de cloreto de sódio, nitratos e nitritos contidos em alimentos defumados, alto consumo de amido e frituras, entre outros, assim como dois fatores de risco bastante presente nas mesas da população amazônica, como as altas concentrações de cloreto de sódio e grande consumo de amido (TEIXEIRA JBA, NOGUEIRA MS, 2008). Mas, mesmo com problemas gastrointestinais, apenas alguns pacientes infectados desenvolvem uma doença grave, como úlcera péptica (10\% -15\%) ou Câncer Gástrico (CG) (1\% -3\%) (SUERBAUM S, MICHETTI P, 2002).

Um estudo realizado por Resende AL et al. (2006) sobre a mortalidade por câncer gástrico no estado do Pará, nos anos de 1980 a 1997, revela as principais causas e magnitude neste estado. Assim segundo tal estudo, o consumo alimentar predominante na população paraense que vivia nas cidades, em grande parte do século passado, reunia algumas características potencialmente favorecedoras do processo de carcinogênese gástrica, destacando-se o elevado consumo de alimentos salgados e de carboidratos, representados pela farinha de mandioca e a ingestão reduzida de legumes e hortaliças.

Por tanto, a adoção da temática justifica-se pelo fato da população residente na região amazônica possuir certa predisposição para o câncer de estômago pelas características alimentares e o estilo de vida, pelo fato dessa patologia estar em ascensão na região, e também pela alta incidência na clínica cirúrgica do hospital 
escolhido para a pesquisa. A pesquisa objetivou definir os principais fatores de risco ambientais presentes nos pacientes internados em um hospital de referência em oncologia de Belém-PA, relacionando-os com peculiaridades regionais amazônicas e com a infecção pela Helicobacter pylori (H. pylori).

\section{MÉTODOS}

Pesquisa do tipo descritiva de corte transversal com uma abordagem quantitativa. Realizado na clínica cirúrgica e no setor da quimioterapia do Hospital Ophir Loyola, Belém - PA. A população amostral foi composta por 32 pacientes internados na clínica cirúrgica do hospital assim como no setor de quimioterapia, que tiveram diagnóstico definido de câncer de estômago, como a presença de resultado de histopatológico em seu prontuário, ou internaram com Autorização de Internação Hospitalar (AIH) para realização de Gastrectomia (cirurgia para retirada de estômago) e vieram a ter diagnóstico definido ao longo da internação. Foram selecionados de maneira aleatória todos os pacientes que entraram para internação e/ou que estavam realizando quimioterapia ambulatorial, no período de janeiro a fevereiro de 2018.

Segue como critério de inclusão ter idade maior que 18 anos, internados com diagnóstico de câncer de estômago definido ou que se defina ao longo da internação, que não tenham patologias secundárias que prejudiquem a comunicação e/ou andamento de maneira satisfatória do estudo, como por exemplo demências, retardos mentais, entre outras, e que aceitaram de maneira voluntária participar da pesquisa, assinando o Termo de Consentimento Livre e Esclarecido. As abordagens dos informantes aconteceram no ambiente de internação ou no próprio ambulatório onde estava sendo realizada a administração dos quimioterápicos. Foi utilizado como instrumento de coleta de dados um questionário pré-estruturado com perguntas fechadas. O questionário foi aplicado em forma de entrevista, onde o próprio pesquisador deu seguimento nas perguntas.

O instrumento foi composto primeiramente de questões que auxiliaram na identificação do perfil sociodemográfico, como a naturalidade e procedência; sexo; idade; renda familiar mensal; estado civil; escolaridade e profissão/ocupação. No segundo momento composta de questões que auxiliaram na identificação dos fatores de risco ambientais, como a ocorrência da infecção pela $H$. pylori; o consumo de bebida alcoólica e tabagismo; prática regular de exercício físico; conservação dos alimentos e perguntas com relação a variados tipos de alimentos que integram a dieta dos usuários. Vale ressaltar que antes de dar início às perguntas 0 pesquisador enfatizou que as respostas deveriam ser embasadas no estilo de vida que 0 informante tinha antes do diagnóstico ser definido. A amostra foi analisada através de estatística descritiva, através de medidas de frequência (absoluta e relativa), tendência central (média aritmética) e variância (desvio padrão). Para análises intragrupos foi utilizado o teste do Qui-quadrado de aderência. Para estimativa de fatores de risco foi utilizado o teste de Regressão Logística Múltipla, com estimativa de Odds ratio. Toda a inferência estatística foi calculada utilizando o software BioEstat 5.4 , adotando $p$-valor significativo $\leq 0.05$. A pesquisa foi avaliada e aprovada em comitê de ética sobre número de CAAE: 77168917.8.0000.5172.

\section{RESULTADOS}

A análise dos dados foi realizada em dois momentos. Primeiramente, levantaram-se dados estatísticos referentes aos dados pessoais dos clientes, como sexo, procedência, idade, escolaridade e renda familiar. No segundo momento analisamos os hábitos e costumes, assim como dados importantes para a pesquisa, como os vícios, a prática de exercício físico, hábitos dietéticos e os meios de conservação de alimentos.

Como apresentado na Tabela 1, cerca de $78 \%$ (46,87\% faixa etária de 51 a 60 anos e 31,37 \% faixa etária 61 a 70 anos) dos informantes apresentavam idade maior que 50 anos de idade. Com relação ao sexo não observamos um valor significativo, obtendo resultados bem similares, 56,25\% masculino e 43,75 \% feminino. Em sua maioria eram pessoas casadas $46,87 \%$, obtendo valores equiparados nas demais variáveis relacionado ao estado civil. $25 \%$ apresentaram menos que 1 salário mínimo de renda e $65,25 \%$ entre 1 salário mínimo e 3 salários de renda mensal. $56,25 \%$ da amostra tinham apenas o ensino fundamental e $6,25 \%$ eram analfabetos, aqueles que concluíram o ensino médio representaram $31,37 \%$ da amostra. 
Tabela 1 - Características sociodemográficas de pacientes de câncer gástrico, Belém, Pará, 2018.

\begin{tabular}{|c|c|c|c|}
\hline Características Sociodemográficas & $\mathbf{N}$ & $\%$ & p-valor \\
\hline \multicolumn{4}{|l|}{ Sexo } \\
\hline Masculino & 18 & 56,25 & \multirow[t]{3}{*}{0.7003} \\
\hline Feminino & 14 & 43,75 & \\
\hline Total & 32 & 100,00 & \\
\hline \multicolumn{4}{|l|}{ Faixa etária } \\
\hline 18 a 35 anos & 2 & 6,25 & \multirow[t]{7}{*}{0.0064} \\
\hline 36 a 50 anos & 4 & 12,50 & \\
\hline 51 a 60 anos & 15 & 46,87 & \\
\hline 61 a 70 anos & 10 & 31,37 & \\
\hline Maior que 70 anos & 1 & 3,12 & \\
\hline Média \pm Desvio Padrão & \multicolumn{2}{|c|}{$54.81 \pm 12.76$} & \\
\hline Total & 32 & 100,00 & \\
\hline \multicolumn{4}{|l|}{ Estado Civil } \\
\hline Solteiro & 8 & 25,00 & \multirow[t]{6}{*}{0.0046} \\
\hline Casado & 15 & 46,87 & \\
\hline União estável & 2 & 6,25 & \\
\hline Divorciado & 2 & 6,25 & \\
\hline Viúvo & 5 & 15,62 & \\
\hline Total & 32 & 100,00 & \\
\hline \multicolumn{4}{|l|}{ Procedência } \\
\hline Região Metropolitana de Belém & 18 & 56,25 & \multirow[t]{3}{*}{0.4414} \\
\hline Interior do Estado & 14 & 43,75 & \\
\hline Total & 32 & 100,00 & \\
\hline \multicolumn{4}{|l|}{ Escolaridade } \\
\hline Analfabeto & 2 & 6,25 & \multirow[t]{5}{*}{0.0016} \\
\hline Ensino Fundamental & 18 & 56,25 & \\
\hline Ensino Médio & 10 & 31,37 & \\
\hline Ensino Superior & 2 & 6,25 & \\
\hline Total & 32 & 100,00 & \\
\hline \multicolumn{4}{|l|}{ Renda familiar } \\
\hline Menor que 1 salário mínimo & 8 & 25,00 & \multirow[t]{6}{*}{$<0.0001$} \\
\hline 1 a 3 salários mínimos & 21 & 65,62 & \\
\hline 4 a 5 salários mínimos & 2 & 6,25 & \\
\hline 6 a 7 salários mínimos & - & - & \\
\hline Maior que 7 salários mínimos & 1 & 3,12 & \\
\hline Total & 32 & 100,00 & \\
\hline
\end{tabular}

Fonte: Questionários da pesquisa, 2019. 
A Tabela 2 apresenta as características clínicas dos pacientes pesquisados e demonstra que a presença da $H$. pylori está em $53,12 \%$ da amostra, estando ausente em $46,87 \%$ dos casos. O tipo histológico adenocarcinoma gástrico é o de maior prevalência para o câncer gástrico, apresentando-se em 96,87 \% dos informantes, apenas 1 (um) paciente apresentou câncer gástrico do tipo linfoma representando 3,12\%.

Tabela 2 - Características clínicas de pacientes de câncer gástrico, Belém, Pará, 2018.

\begin{tabular}{llll}
\hline Características Clínicas & N & $\%$ & p-valor \\
\hline Infecção por Helicobacter pylori & 17 & 53,12 & 0.7003 \\
Presente & 15 & 46,87 & \\
Ausente & 32 & 100,00 & \\
Total & & & \\
Resultado do exame histopatológico & 31 & 96,87 & $<0.0001$ \\
Adenocarcinoma & 1 & 3,12 & \\
Outros tipos histológicos & 32 & 100,00 & \\
Total & &
\end{tabular}

Fonte: Questionários da pesquisa, 2019.

46,87\% dos usuários nunca fumaram e 53,12\% fumaram, porém já haviam parado há algum tempo. 56,25 $\%$ eram etilistas, ficando equiparado com os não etilistas que representaram $43,75 \%$ (Tabela 3). Quase que totalidade do espaço amostral se revelou em conservar seus alimentos na geladeira $(90,62 \%) \mathrm{com} n=29 \mathrm{e}$ apenas 3 usuários conservavam em meios salgados (9,37\%).

Tabela 3 - Fatores de risco em pacientes de câncer gástrico, Belém, Pará, 2018.

\begin{tabular}{lccc}
\hline Fatores de risco & N & $\%$ & p-valor \\
\hline Prática de exercício físico regular & 21 & 67,62 & 0.1237 \\
Sim & 11 & 34,37 & \\
Não & 32 & 100,00 & \\
Total & & & \\
Tabagismo & - & - & 0.7003 \\
Sim & 15 & 46,87 & \\
Não (nunca fumou) & 17 & 53,12 & \\
Não (parou antes do diagnóstico) & 32 & 100,00 & \\
Total & & & \\
Etilismo & 18 & 56,25 & 0.4414 \\
Sim & 14 & 43,75 & \\
Não & 32 & 100,00 & \\
Total & & & \\
Conservação de alimentos & 29 & 90,62 & $<0.0001$ \\
Geladeira & 3 & 9,37 & \\
Meios salgados & 32 & 100,00 & \\
Total & & & \\
\hline
\end{tabular}

Fonte: Questionários da pesquisa, 2019. 
Quanto ao consumo de alimentos industrializados observamos respostas bastante equilibradas em todas as variáveis (Tabela 4). Em sua maioria apresentando maior frequência nas faixas do consumo raro e do consumo menos de 3 vezes por semana. O consumo raro se mostrou maior em enlatados, conservas, presunto/mortadela e defumados $(43,75 \%, n=14 ; 40,62 \%, n=13 ; 43,75 \%, n=14$ e $59,75 \%, n=19$ respectivamente). Salsicha era consumida em sua maioria menos de 3 vezes por semana $(43,75 \%, n=14)$.

Tabela 4 - Consumo de alimentos industrializados por pacientes de câncer gástrico, Belém, Pará, 2018.

\begin{tabular}{|c|c|c|c|}
\hline Consumo de alimento & $\mathbf{N}$ & $\%$ & p-valor \\
\hline \multicolumn{4}{|l|}{ Enlatados } \\
\hline Não consome & 9 & 28,12 & 0.0033 \\
\hline Raramente & 14 & 43,75 & \\
\hline Menos de 3 vezes na semana & 7 & 21,87 & \\
\hline Mais de 3 vezes na semana & - & - & \\
\hline Diariamente & 2 & 6,25 & \\
\hline Total & 32 & 100,00 & \\
\hline \multicolumn{4}{|l|}{ Conserva } \\
\hline Não consome & 5 & 15,62 & 0.0033 \\
\hline Raramente & 13 & 40,62 & \\
\hline Menos de 3 vezes na semana & 11 & 34,37 & \\
\hline Mais de 3 vezes na semana & - & - & \\
\hline Diariamente & 2 & 6,25 & \\
\hline Total & 32 & 100,00 & \\
\hline \multicolumn{4}{|l|}{ Salsicha } \\
\hline Não consome & 7 & 21,87 & 0.0024 \\
\hline Raramente & 10 & 31,25 & \\
\hline Menos de 3 vezes na semana & 14 & 43,75 & \\
\hline Mais de 3 vezes na semana & & - & \\
\hline Diariamente & 1 & 3,12 & \\
\hline Total & 32 & 100,00 & \\
\hline \multicolumn{4}{|l|}{ Presunto/mortadela } \\
\hline Não consome & 7 & 21,87 & 0.0012 \\
\hline Raramente & 14 & 43,75 & \\
\hline Menos de 3 vezes na semana & 10 & 31,28 & \\
\hline Mais de 3 vezes na semana & 1 & 3,12 & \\
\hline Diariamente & - & - & \\
\hline Total & 32 & 100,00 & \\
\hline \multicolumn{4}{|l|}{ Defumados } \\
\hline Não consome & 5 & 15,62 & $<0.0001$ \\
\hline Raramente & 19 & 59,75 & \\
\hline Menos de 3 vezes na semana & 7 & 21,87 & \\
\hline Mais de 3 vezes na semana & 1 & 3,12 & \\
\hline Diariamente & - & - & \\
\hline Total & 32 & 100,00 & \\
\hline
\end{tabular}

Fonte: Questionários da pesquisa, 2019.

O consumo de sal e/ou alimentos processados em sal (Tabela 5), principalmente carne seca salgada, peixes salgados e churrasco se mostraram evidente na faixa de consumo daqueles que consomem mais de 3 vezes na semana e menos de 3 vezes na semana, representando $21,87 \%, 21,87 \%$ e $37,50 \%$ respectivamente (consumo maior de 3 vez por semana) e $37,50 \%, 40,62 \%$ e $21,87 \%$ (menor de 3 vez na semana). Alimentação a base de peixe em conserva foi raro, $n=14-43,75 \%$. Em alimentos fritos foi obtido respostas semelhantes, com maioria respondendo consumo raro, $n=11-34,37 \%$. 
Tabela 5 - Consumo de alimento segundo o modo de preparo/conservação por pacientes de câncer gástrico, Belém, Pará, 2018.

\begin{tabular}{|c|c|c|c|}
\hline Consumo de alimento & $\mathbf{N}$ & $\%$ & p-valor \\
\hline \multicolumn{4}{|l|}{ Carne seca salgada } \\
\hline Não consome & 3 & 9,37 & 0.0004 \\
\hline Raramente & 10 & 31,25 & \\
\hline Menos de 3 vezes na semana & 12 & 37,50 & \\
\hline Mais de 3 vezes na semana & 7 & 21,87 & \\
\hline Diariamente & - & - & \\
\hline Total & 32 & 100,00 & \\
\hline \multicolumn{4}{|l|}{ Peixe salgado } \\
\hline Não consome & 2 & 6,25 & 0.0003 \\
\hline Raramente & 9 & 28,12 & \\
\hline Menos de 3 vezes na semana & 13 & 40,62 & \\
\hline Mais de 3 vezes na semana & 7 & 21,87 & \\
\hline Diariamente & 1 & 3,12 & \\
\hline Total & 32 & 100,00 & \\
\hline \multicolumn{4}{|l|}{ Peixe em conserva } \\
\hline Não consome & 7 & 21,87 & 0.0002 \\
\hline Raramente & 14 & 43,75 & \\
\hline Menos de 3 vezes na semana & 7 & 21,87 & \\
\hline Mais de 3 vezes na semana & 3 & 9,37 & \\
\hline Diariamente & 1 & 3,12 & \\
\hline Total & 32 & 100,00 & \\
\hline \multicolumn{4}{|l|}{ Churrasco } \\
\hline Não consome & - & - & 0.0064 \\
\hline Raramente & 11 & 34,37 & \\
\hline Menos de 3 vezes na semana & 7 & 21,87 & \\
\hline Mais de 3 vezes na semana & 12 & 37,50 & \\
\hline Diariamente & 2 & 6,25 & \\
\hline Total & 32 & 100,00 & \\
\hline \multicolumn{4}{|l|}{ Alimentos fritos } \\
\hline Não consome & 8 & 25,00 & 0.0789 \\
\hline Raramente & 11 & 34,37 & \\
\hline Menos de 3 vezes na semana & 8 & 25,00 & \\
\hline Mais de 3 vezes na semana & 2 & 6,25 & \\
\hline Diariamente & 3 & 9,37 & \\
\hline Total & 32 & 100,00 & \\
\hline
\end{tabular}

Fonte: Questionários da pesquisa, 2019. 
A Tabela 6 vem representando o consumo diário de farinha de mandioca sinalizando mais de $65 \%(n=21)$ e $15,62 \%$ de consumo mais que 3 vezes por semana. Outros alimentos como o tucupi derivado da mandioca não observamos distribuição significativa para efeito estatístico. Com relação ao consumo de frutas cítricas e verduras, fatores considerados protetores, observamos distribuições variadas e semelhantes nas faixas de consumo. Para consumo de frutas temos frequência mais elevada para ingesta menos de 3 vez por semana $(31,25 \%-n=10)$ com leve desvio para consumo raro e consumo zero. Na ingesta de verduras prevaleceu 0 consumo mais de 3 vezes por semana $(31,25 \%-n=10)$.

Tabela 6 - Consumo de alimentos regionais e alimentos protetores por pacientes de câncer gástrico, Belém, Pará, 2018.

\begin{tabular}{llll}
\hline Consumo de alimento & N & $\%$ & p-valor \\
Farinha de mandioca & & & $<0.0001$ \\
Não consome & 1 & 3,12 & \\
Raramente & 3 & 9,37 & \\
Menos de 3 vezes na semana & 2 & 6,25 & \\
Mais de 3 vezes na semana & 5 & 15,62 & \\
Diariamente & 21 & 65,62 & 0.0231 \\
Total & 32 & 100,00 & \\
Tucupi derivado da mandioca & & & \\
Não consome & 9 & 28,12 & \\
Raramente & 13 & 40,62 & \\
Menos de 3 vezes na semana & 5 & 15,62 & \\
Mais de 3 vezes na semana & 2 & 6,25 & \\
Diariamente & 3 & 9,37 & \\
Total & 32 & 100,00 & \\
Frutas cítricas & & & \\
Não consome & 4 & 12,50 & \\
Raramente & 8 & 25,00 & \\
Menos de 3 vezes na semana & 10 & 31,25 & \\
Mais de 3 vezes na semana & 5 & 15,62 & \\
Diariamente & 5 & 15,62 & \\
Total & 32 & 100,00 & \\
Verduras & & & \\
Não consome & 4 & 12,50 & \\
Raramente & 6 & 18,75 & \\
Menos de 3 vezes na semana & 8 & 25,00 & \\
Mais de 3 vezes na semana & 10 & 31,25 & \\
Diariamente & 4 & 12,50 & \\
Total & 32 & 100,00 & \\
\hline Font: Questonánios da pesqusa, & & & \\
\end{tabular}

Fonte: Questionários da pesquisa, 2019.

\section{DISCUSSÃO}

O câncer gástrico é um dos seis tumores malignos mais frequentes no Brasil, entre os homens e mulheres, e em ambos os gêneros, a incidência aumenta a partir de 35-40 anos (ZILBERSTEIN B et al., 2013). Em concordância com demais estudos, nesta pesquisa podemos afirmar que a idade elevada pode ser considerada como um fator de risco para o adenocarcinoma gástrico. Como apresentado na Tabela 1, cerca de $78 \%$ (46,87 faixa etária de 51 a 60 anos e 31,37\% faixa etária 61 a 70 anos) dos informantes apresentavam idade maior que 50 anos de idade ( $p$ - 0.0064).

Estudos retrospectivos em uma análise de subgrupos identificaram a idade como uma variável prognóstica significativa, o que corrobora a perspectiva da atual pesquisa, a qual ressalta o maior risco da doença em 
idades mais avançadas, enquanto que em outro estudo, a influência da idade não era de valor prognóstico independente, este estudo determinou que a sobrevivência foi determinada pelo estágio e completude da ressecção. (YOKOTA T et al, 2000; KIM TH et al., 2000; MEDINA-FRANCO H et al., 2000).

Enfatizando o estudo de Medina-Franco $\mathrm{H}$ et al (2000) ao afirmar que nem a idade nem o gênero mostraram-se definitivamente de importância prognóstica para a morte por câncer recorrente ou metastático, em nosso estudo não chegamos em um valor de significância considerável com relação a variável sexo ( $p$ 0.7003), não se demonstrando como um fator de risco.

No geral, podemos afirmar que a baixa escolaridade e renda são fatores de risco importantes para a carcinogênese gástrica ( $p-0.0016$ e $<0.0001$ respectivamente), demonstrando que mais de $56,25 \%$ da amostra apresentam apenas o ensino fundamental e 6,25\% são analfabetos, assim como $25 \%$ apresentam menos que 1 salário mínimo de renda e $65,25 \%$ entre 1 salário e 3 salários de renda salarial mensal. A variável se revelou como um fator de risco $(\mathrm{p}-0.4414)$, estando a percentagem similares entre as pessoas procedentes do interior e da capital do Pará.

O CG (Câncer Gástrico) revelou-se inversamente relacionado ao status socioeconômico, de modo que a alta posição socioeconômica está associada a um risco reduzido de CG, particularmente cardia e subtipos intestinais (NAGEL G et al., 2007). As profissões que correm maior risco de CG são pescadores, operadores de máquinas, enfermeiros, cozinheiros, lavadores e em tinturarias, uma vez que as principais exposições ocupacionais incluem poeira, óxidos de nitrogênio, compostos $\mathrm{N}$-nitroso e radiação (ARAGONES $\mathrm{N}$ et al., 2002).

De acordo com o estudo de Rugge M (2015) e demais estudos em todo o mundo, a infecção pela $H$. pylori se apresenta como um importante fator de risco ambiental para a ocorrência de câncer gástrico, principalmete relacionado ao adenocarcinoma, porém, neste estudo não podemos observar um valor de significância considerável para tal realidade.

A Tabela 2 apresenta as características clínicas dos pacientes pesquisados e demonstra que a presença da $H$. pylori está em apenas $53,12 \%$ ( $p-0.7003$ ) da amostra, estando ausente em $46,87 \%$ dos casos, impactando com os estudos citados e não favorecendo a um fator de risco, no entanto, Warren JR, Marshall B (1983) descobriram a associação entre H. pylori e gastrite em 1982. Em 1994, H. pylori foi classificado como um carcinógeno de classe I pela Agência Internacional de Pesquisa sobre Câncer. Em seguida, foi acusado de ser o principal fator ambiental que causa CG (FORMAN D et al, 1991; PARSONNET J et al., 1991; IARC, 1994). A infecção por $H$. pylori é uma causa comum, mas na atual pesquisa de incidência de câncer o fator mais relacionado a doença associa-se de maneira mais íntima aos hábitos alimentares, que na região norte torna-se um fator cultural e social, caracterizado pelo consumo da mandioca e seus derivados.

Na população em geral, a infecção por $\mathrm{H}$. pylori atinge $60 \%$, mas em pacientes com $\mathrm{CG}$, é mais comum (84\%) ou mesmo inevitável (CG não cardíaco) (PARSONNET J et al., 1991; GONZÁLEZ CA, 2012). A correlação entre infecção por $H$. pylori e GC relaciona-se também com idade mais nova ( $<40$ anos) e está envolvida nos tipos intestinal e difuso de CG de não cardia. $O$ último é mais comum no câncer gástrico de início precoce (EOGC) (RUGGE M et al, 1999; CORREA P, HOUGHTON J, 2007).

Podemos afirmar sem dúvida que o tipo histológico adenocarcinoma gástrico é o de maior prevalência para o câncer gástrico, apresentando-se em $96,87 \%$ dos informantes $(p<0.0001)$.

Muitos estudos confirmaram que o tabagismo aumenta o risco de $C G$, ambos os subtipos cárdia e não cárdia (NISHINO Y et al, 2006; LADEIRAS-LOPES R et al, 2008). Foi demonstrado que o risco de CG é aumentado em $60 \%$ em homens e $20 \%$ em mulheres fumantes em comparação com não fumantes. $O$ risco de CG é menor nos antigos fumantes em comparação com fumantes ocasionais, e os fumantes com maior consumo de cigarros (> 20 cigarros por dia) estão em maior risco de CG (LADEIRAS-LOPES R et al, 2008).

O consumo de álcool também predispõe o CG. A associação entre abuso de álcool e câncer de cárdia gástrica foi relatada (LAGERGREN J et al, 2000). 
Mais de $90 \%$ da população amostral conservava seus alimentos em geladeira, demonstrando que o tipo de conservação dos alimentos não é considerado um fator de risco para o adenocarcinoma gástrico na região amazônica $(p<0.0001)$. No entanto, a diminuição geral da incidência de CG pode ser explicada por padrões mais elevados de higiene, conservação melhorada dos alimentos, alta ingestão de frutas e vegetais frescos e pela erradicação de H. pylori (MUNOZ N e FRANCESCHI S, 1997).

Os nitratos têm ampla distribuição na natureza, ocorrem em plantas, são usados na preservação de alimentos. Os nitratos estão principalmente associados a substâncias industrializadas e representam um importante fator de risco ambiental para a carcinogenese gástrica (RAMON JM et al., 1993). Neste estudo, como podemos observar na Tabela 4, essas substâncias não se revelaram como um importante fator de risco ambiental, já que a maioria das respostas estavam enquadradas na faixa do consumo raro ou menos que 3 vezes por semana, no qual consideramos para efeito de significância as respostas nas faixas de mais que 3 vezes na semana ou o consumo diário.

Em um estudo caso-controle, Ramon JM et al (1993), dietas identificadas ricas em sal, alimentos defumados ou mal conservados, nitratos, nitritos e aminas secundárias para serem associados a um risco aumentado de câncer gástrico. Acredita-se que a associação resulte do consumo prolongado excessivo de alimentos salgados ou em conserva, o que leva a gastrite atrófica e uma alteração no ambiente gástrico com a geração de compostos $\mathrm{N}$-nitroso cancerígenos.

Neste estudo, descobrimos que o consumo de sal em alimentos processados, em especial carne seca salgada, peixe salgado e churrasco, foram associados a um risco aumentado de câncer gástrico $(p-0.0004$, 0.0003 e 0.0064 respectivamente) - Tabela 5.

Mesmo que em alguns momentos a resposta se concentrava na faixa de consumo menos que 3 vezes por semana, observamos em sua maioria respostas relacionadas com o consumo diário ou maior que 3 vezes por semana, além de confirmar que o consumo raro ou a ausência de consumo foram poucos demonstrados. Esses resultados forneceram alguma evidência de que alimentos salgados possam contribuir ao alto risco de câncer gástrico na área de estudo.

Para investigar a associação entre alimentos processados com sal e câncer gástrico, um estudo de casocontrole baseado em hospital foi conduzido em uma área de alto risco da China. Cento e sete casos recémdiagnosticados com confirmação histológica de câncer gástrico e 209 controles foram recrutados. A informação sobre a ingestão dietética foi coletada com um questionário validado de frequência alimentar.

A comparação do grupo de ingestão alta com o consumo nunca mais de alimentos processados com sal, carne salgada, vegetais em conserva e vegetais preservados foi significativamente associada ao aumento do risco de câncer gástrico. Entretanto, a preferência do sabor do sal na dieta mostrou uma relação doseresposta com câncer gástrico (LIN SH et al., 2014).

Os resultados sugerem que o consumo de alimentos de origem regional, principalmente o consumo diário de farinha de mandioca $(p<0.0001)$ representando mais de $65 \%$ e $15,62 \%$ de consumo mais que 3 vezes por semana, estão positivamente associados ao câncer gástrico (Tabela 6). Outros alimentos como o tucupi derivado da mandioca não se apresentou como fator de risco já que a distribuição ficou equiparada dentro das variadas opções de respostas dos participantes da pesquisa.

Com relação aos fatores protetores para o adenocarcinoma como o consumo de frutas cítricas e de vegetais variados, não podemos afirmar que teve relação positiva como um fator protetor ( $p-0.9154$ e 0.7221 respectivamente), pois as respostas dos pacientes ficaram distribuídas de maneira equilibradas não provocando um valor significante para pesquisa.

A influência benéfica da dieta rica em vitaminas parece ser particularmente notável no caso de deficiência de folato e selênio (CAMARGO MC et al., 2012).

Os resultados de vários estudos sobre essas propriedades são ainda incoerentes (JENAB M et al., 2006). Portanto, o problema requer novas investigações. 


\section{CONCLUSÃO}

A carcinogênese é um processo complexo para o qual contribuem tanto fatores predisponentes intrínsecos (predisposição herdada), quanto extrínsecos (fatores de risco ambientais). Verificou-se que os clientes com diagnóstico de câncer gástrico atribuíram o surgimento da doença a algum fator, mencionando fatores de risco para a mesma. Observamos que, mesmo com maior número de citações de uso raro ou o uso inferior a três vezes por semana de alimentos considerados de risco, a ingesta de alimentos considerados protetores também se fazia de maneira satisfatória nos hábitos alimentares de nossa amostra. Em sua maioria conservavam os alimentos na geladeira. Eram pessoas de baixa renda e nível de escolaridade também baixo. O consumo de alimentos processados em sal se mostrou positivo para a ocorrência do adenocarcinoma, além de definirmos alguns fatores de risco para a infecção do $H$. pylori. Podemos afirmar que há alimentos que foram mais consumidos e indicam a maior predisposição ao câncer e que já foram citados anteriormente. A identificação destes fatores é importante para que se possam programar ações preventivas para diminuir a incidência de tumores de localização gástrica em áreas e países em que ela ainda é alta, como é o caso do Brasil, principalmente a região amazônica.

\section{REFERÊNCIAS}

1. ARAGONES N, et al. Stomach cancer and occupation in Sweden. Occup Environ Med. 2002; 59(5): $329-37$.

2. CAMARGO MC, et al. Plasma selenium measurements in subjects from areas with contrasting gastric cancer risks in Colombia. Arch Med Res. 2008; 39(4): 443-51.

3. CORREA P, HOUGHTON J. Carcinogenesis of Helicobacter pylori. Gastroenterology. 2007; 133(2): 659-72.

4. FORMAN D, et al. Association between infection with Helicobacter pylori and risk of gastric cancer: evidence from a prospective investigation. BMJ. 1991; 302(6788): 1302-5.

5. GONZALEZ CA, et al. Fruit and vegetable intake and the risk of gastric adenocarcinoma: A reanalysis of the European prospective investigation into cancer and nutrition (EPIC-EURGAST) study after a longer follow-up. Int. J. Cancer. 2012; 131(12): 2910-9.

6. IARC. Working Group on the Evaluation of Carcinogenic Risks to Humans 1994; 69: 1-631.

7. IMOBERDORF R, et al. Cancer and nutrition - A paradigma shift. Laryngorhinootologie. 2017; 96(8): 514-518.

8. JENAB M, et al. Plasma and dietary carotenoid, retinol and tocopherol levels and the risk of gastric adenocarcinomas in the European prospective investigation into cancer and nutrition. Br J Cancer. 2006; 95(3): 406-15.

9. KIM TH, et al. Perigastric lymph node status can be a simple prognostic parameter in patients with gastric cancer. Hepatogastroenterology. 2000; 47(35): 1475-8.

10. LADEIRAS-LOPES R, et al. Smoking and gastric cancer: systematic review and meta-analysis of cohort studies. Cancer Causes Control. 2008; 19(7): 689-701.

11. LAGERGREN J, et al. The role of tobacco, snuff and alcohol use in the aetiology of cancer of the oesophagus and gastric cardia. Int J Cancer. 2000; 85(3): 340-6.

12. LIN SH et al. Salt processed food and gastric cancer in a Chinese population. Asian Pac J Cancer Prev. 2014; 15(13): 5293-8.

13. MANNISTO S, et al. Dietary patterns and risk of breast cancer: results from three cohort studies in the DIETSCAN project. Control of Causes of Cancer. 2015; 16(6): 725-33.

14. MEDINA-FRANCO $\mathrm{H}$, et al. Clinicopathological characteristics of gastric carcinoma in young and elderly patients: a comparative study. Ann Surg Oncol. 2000; 7(7): 515-9.

15. MUNOZ N, FRANCESCHI S. Epidemiology of gastric cancer and perspectives for prevention. Salud Publica Mex. 1997; 39(4): 318-30.

16. NAGEL G, et al. Socioeconomic position and the risk of gastric and oesophageal cancer in the european prospective invéstigation into cancer and nutrition (EPIC-EURGAST). Int J Epidemiol. 2007; 36(1): 66-76.

17. NISHINO Y, et al. Research Group for the Development and Evaluation of Cancer Prevention Strategies in Japan Tobacco smoking and gastric cancer risk: an evaluation based on a systematic review of epidemiologic evidence among the Japanese population. Jpn J Clin Oncol. 2006; 36(12): 800-7.

18. PARSONNET J, et al. Helicobacter pylori infection and the risk of gastric carcinoma. N Engl J Med. 1991; 325(16): 1127-31.

19. RAMON JM, et al. Fatores dietéticos e risco de câncer gástrico. Um estudo caso-controle em Espanha. Câncer. 1993; 71: 17311735.

20. RESENDE AL, et al. Dieta e câncer gástrico: aspectos históricos associados ao padrão de consumo alimentar no estado do Pará. Revista de Nutrição, Campinas, jul./ago., 2006; 19(4): 511-519.

21. RUGGE M, et al. Patients younger than 40 years with gastric carcinoma: Helicobacter pylori genotype and associated gastritis phenotype. Cancer. 1999; 85(12): 2506-11.

22. SUERBAUM S, MICHETTI P. Helicobacter pylori infection. N Engl J Med. 2002; 347(15): $1175-86$.

23. TEIXEIRA JBA, NOGUEIRA MS. Câncer gástrico: fatores de risco em clientes atendidos nos serviços de atenção terciária em um município do interior paulista. Revista Latino-americana de Enfermagem. 2008; 11: 43-8.

24. WARREN JR, MARSHALL B. Unidentified curved bacilli on gastric epithelium in active chronic gastritis. Lancet. 1983; 1(8336): $1273-5$.

25. YOKOTA T, et al. Significant prognostic factors in patients with early gastric cancer. Int Surg. 2000; 85(4): 286-90.

26. ZILBERSTEIN B, et al. Consenso brasileiro sobre câncer gástrico: diretrizes para o câncer gástrico no Brasil. ABCD, arq. bras. cir. dig., 2013; 26(1): 2-6. 